\title{
FADING PHENOMENA OF HF-WAVES FROM STANDARD FREQUENCY AND TIME SIGNAL STATIONS [ I ]
}

\author{
Sazanami Yano \\ Department of Electrical Engineering \\ Kochi National College of Technology \\ Monobe, Nankoku, Kochi 783, Japan \\ Akira Ishimaru \\ Department of Electrical Engineering, FT-10 \\ University of Washington \\ Seattle, Washington 98195, USA \\ Toshio Ogawa \\ Science Laboratory International \\ 2c, Sunset Building, 1510-1 Kamobe, Kochi 780, Japan
}

\begin{abstract}
High frequency radio waves transmitted from standard frequency and time signal stations such as JJY and BPM have deep fading near dawn. The fading is caused by the interference of two or more waves which consist of upper rays and lower rays, or right-handed and left-handed polarization waves, or all of these waves under a special condition. The trace on a sonagram of these waves received during the fading shows a $\mathrm{C}$-shaped form when increasing of the ionospheric electron density and the inversed $\mathrm{C}$-shaped form when decreasing of the ionospheric electron density. In this report, we discuss theoretically why this $\mathrm{C}$-shaped form appears in the frequency, and give a calculation method to interpret the physical mechanism of this kind of phenomena. Theoretically derived dynamic spectra are consistent with traces on the sonagram obtained by experiments.
\end{abstract}

\section{Introduction}

Observations of ionospheric disturbances have been made actively in recent years using the Doppler effect of the standard frequency and time signals. This Doppler method is useful because a small disturbance can be detected by a simple device. The observations and study of such HF Doppler effect are being made continuously at more than ten locations in Japan. This had been one of the targetted subjects of the Middle Atmosphere Program (MAP). We had been participating in the observation network since 1979 to receive the standard frequency and time signals of JJY (Nazaki), BPV (Shanghai), BPM (Xian), etc.

Our observation revealed that the fading of the waves and the variation in the $\mathrm{C}$-shaped and the inversed $\mathrm{C}(\supset)$-shaped forms of the traces on the sonagrams were caused by the Doppler deviation of waves which appear near dawn. This variation in frequency depends greatly on the propagation distance. A similar frequency variation in the $\mathrm{C}$-shaped characteristic was observed also at Boulder (Davies, 1965) and Fukuoka (Kitamura et al., 1979; 1980). Kitamura et al. $(1979 ; 1980)$ and Takefu et al. (1982) analyzed this phenomenon by using the full wave method.

We proposed an analysis method using a geometrical optics approximation to our observed results and found that the calculated results in the followings closely coincided with the measured values (Yano et al., 1982a; 1982b; 1983): (1) the theoretically derived time-intervals between the C-shaped traces of the waves from three stations distributed over a wide area in Japan and China, (2) relative bearing of the $\mathrm{C}$-shaped and inversed $\mathrm{C}$-shaped characteristics, and (3) the variation in the lower slopes of the $\mathrm{C}$-shaped characteristic. In this paper, we discuss the analysis method to interpret the fading, the $\mathrm{C}$-shaped and inversed $\mathrm{C}$-shaped characteristics, and compare the calculated traces with the measured traces on the sonagram.

\section{Measured spectral traces on sonagrams}

We received the waves from the standard frequency and time signal stations called by JJY, BPM, and BPV shown in Fig.1. In the figure, the locations of the stations and of the ionospheric reflection points of the waves are indicated by labelling latitude and longitude. The block diagram of the receivers is shown in Fig.2. In the figure, the abbreviation STD means the standard crystal oscillator keeping the frequency stability with factor of $10^{-10}$, and RTA means a real time frequency analyzer. In the figure, the multiplier and divider in the local oscillator satisfy Eq.(1) 
and the frequency of the output signal is given by Eq.(2).

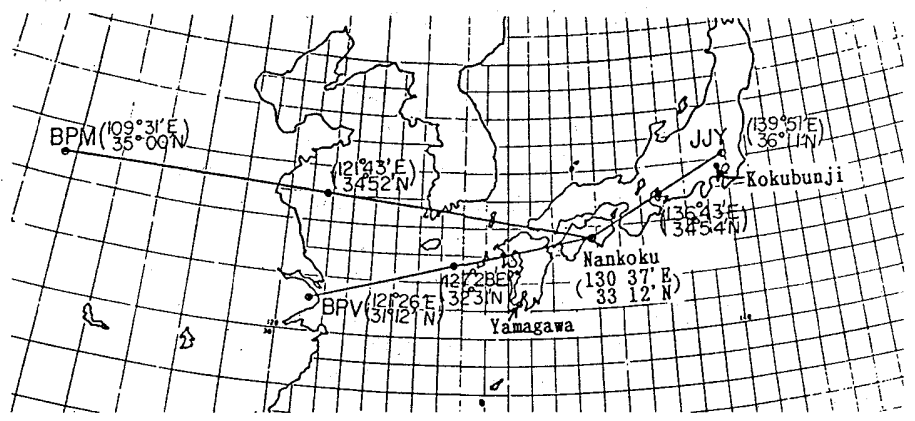

Fig.1. Locations of the stations transmitting the standard frequency and time signal and of the ionospheric reflection points.

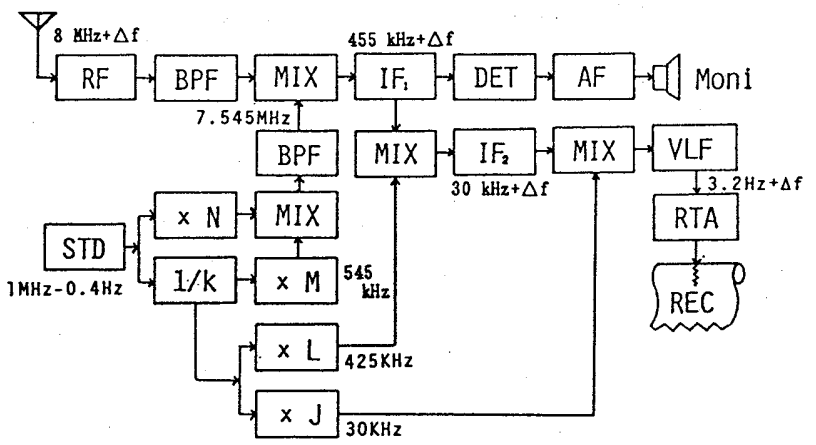

Fig.2. Block diagram of the receiver. The frequencies indicated by the block are for the signal frequency of $8 \mathrm{MHz}$.

$$
\begin{aligned}
& f-f_{s}\left(N+\frac{M+L+J}{K}\right)=0 \\
& f_{0}=\Delta f_{S}\left(N+\frac{M+L+J}{K}\right)+\Delta f
\end{aligned}
$$

where $f$ is the receiving frequency with Doppler deviation of $\triangle f$, and $f_{s}$ is the frequency of the local oscillator with a shift of $\triangle f_{\mathrm{s}}$. We use $N=\left(f-f_{\mathrm{s}}\right) \times 10^{-6}, \mathrm{f}_{\mathrm{s}}=1 \mathrm{MHz}, \triangle \mathrm{f}_{\mathrm{z}}=0.4 \mathrm{~Hz}, \mathrm{~K}=200, \mathrm{M}=109$, $\mathrm{L}=85$, and $\mathrm{J}=6$ to avoid image interferences for each local frequency and to get a wide dynamic amplitude range of the input voltage. An inverted $V$ antenna was used for the receiving antenna.

Figures $3($ a) $\sim$ (d) show examples of measured sonagrams. (a) shows C-shaped dual traces from three stations called JJY, BPM, and BPV, with superposed trace by two hopped waves. (b) shows $\mathrm{C}$-shaped trace with a sharp nose made by JJY signal. (c) shows inversed C-shaped traces labeled $A$ and $B$ which are on 10 and $5 \mathrm{MHz}$ traces of BPM, respectively. (d) shows $\infty$-shaped trace in BPM $10 \mathrm{MHz}$. This is also a kind of inversed $\mathrm{C}$-shaped trace. The mark $\mathrm{A}$ shows a nose point and $\mathrm{P}$ shows a cross over point.

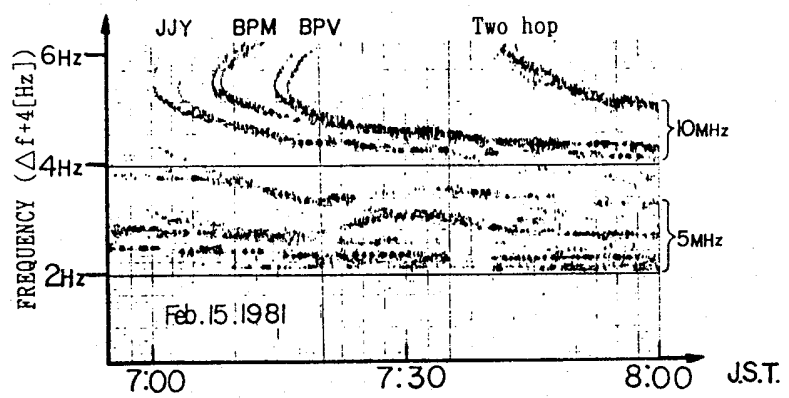

(a) 


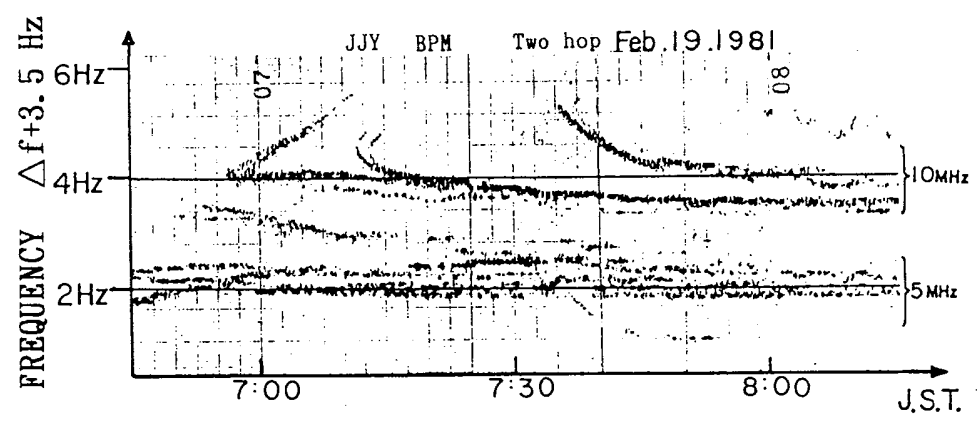

(b)

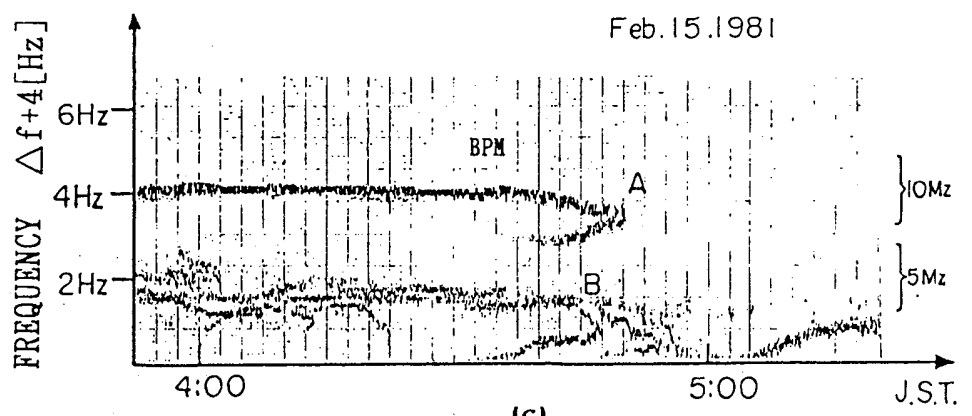

(c)

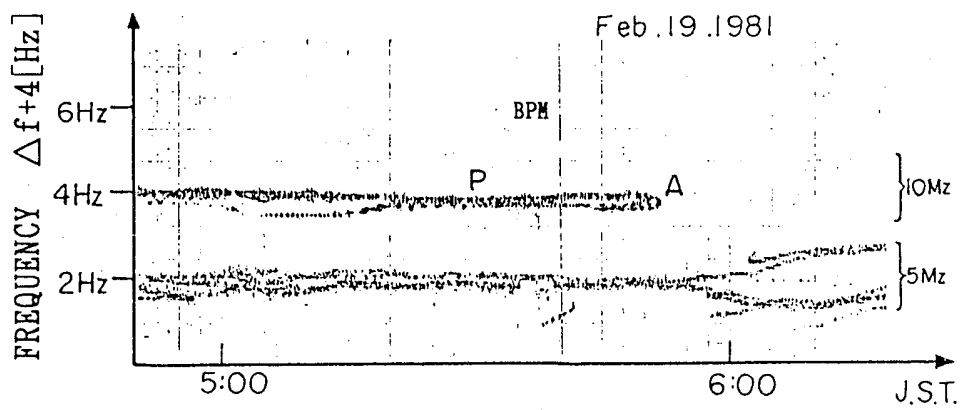

(d)

Fig.3. Examples of measured sonagrams. (a) C-shaped dual traces from three stations. (b) C-shaped trace with a sharp nose made by JJY signal. (c) Inversed C-shaped traces labeled $A$ and $B$ which are on 10 and $5 \mathrm{MHz}$ traces of BPM, respectively. (d) $\infty$-shaped trace in BPM $10 \mathrm{MHz}$. The mark $A$ shows a nose point and $P$ shows a cross over point.

In Figs.l and 3, the station called BPV in Shanghai for the standard frequency and time signal was operated on days when present data were obtained, but it was closed a few days later and was taken the place by BPM station in Xian. BPV and BPM were observed simultaneously for about one month before the closing of BPV.

\section{Derivation of propagation path}

Taking a flat surface approximation for the ground and the ionosphere (Budden, 1961), we use the coordinate system as shown in Fig.4(a). In this figure, the origin of the coordinates and the point of $D$ are the source point and the receiving point, respectively. $h_{1}$ and $h_{0}$ are the height of the lower boundary of the ionosphere and the maximum electron density, respectively. In addition, supposing a parabolic profile for the electron density distribution in the ionosphere, we get Eq.(3).

$$
N(z, t)=N_{0}(t)\left\{1-\left(\frac{h_{0}-Z}{h_{0}-h_{1}}\right)^{2}\right\}
$$


The electron density profiles are shown in Fig.4(b) at the times; $t_{1}, t$, and $t_{2}$, where the relation $t_{1}<t<t_{2}$ holds during the increasing phase of the electron density, and $t_{1}>t>t_{2}$ during the decreasing phase.

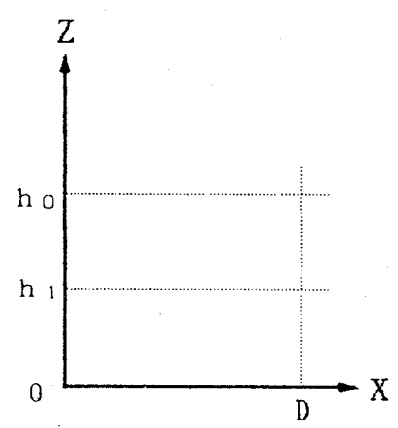

(a)

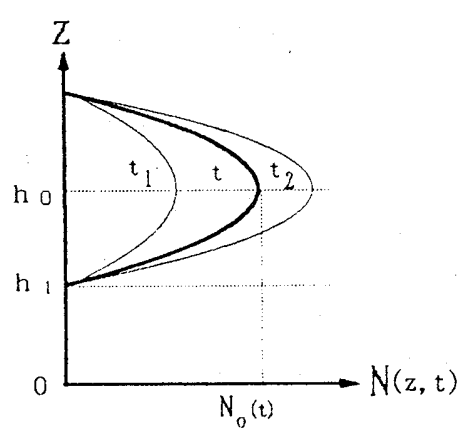

(b)

Fig.4. Coordinate system and electron density profile. (a) Flat ground model. The points $O$ and $D$ show a source and a receiver point, respectively. (b) Parabolic profile of the electron density distribution in the ionosphere. Refer to the text for other symbols.

In Fig. $4(\mathrm{~b}), \mathrm{N}(\mathrm{z}, \mathrm{t})$ is the electron density which is the function of the height $\mathrm{z}$ and time $\mathrm{t}$, and $\mathrm{N}_{\mathrm{o}}(\mathrm{t})$ is the maximum electron density at the time $t$.

Assuming a lossless plasma for the ionosphere, the square of refractive index is given by Eq. (4) which is gotten from Appleton-Hartree's formula with a quasi-longitudinal approximation (Budden, 1961a; Davies, 1965).

$$
\mathrm{n}^{2}=1-\frac{\mathrm{X}}{1 \pm \mathrm{Y}_{\mathrm{L}}}, \quad \mathrm{X} \equiv \frac{\omega_{\mathrm{p}}}{\omega}, \quad \mathrm{Y}_{\mathrm{L}} \equiv \frac{\omega_{\mathrm{C}}}{\omega}
$$

where $\omega_{\mathrm{p}}$ and $\omega_{\mathrm{c}}$ are the angular electron plasma and the angular erectron cyclotron frequencies, respectively. The angular plasma frequency depends upon electron density in the ionosphere as given by Eq.(5).

$$
\begin{aligned}
& \omega_{\mathrm{p}}^{2}=\frac{\mathrm{e}^{2} \mathrm{~N}(\mathrm{z}, \mathrm{t})}{\mathrm{m} \varepsilon_{0}}=\omega_{\mathrm{pO}}^{2}(\mathrm{t})\left\{1-\left(\frac{\mathrm{h}_{\mathrm{O}}-\mathrm{Z}}{\mathrm{h}_{\mathrm{O}}-\mathrm{h}_{1}}\right)^{2}\right\} \\
& \omega_{\mathrm{p} O}^{2}=\frac{\mathrm{e}^{2}}{\mathrm{~m} \varepsilon_{0}} \mathrm{~N}_{0}(\mathrm{t})
\end{aligned}
$$

where $e$ and $m$ are the charge and mass of an electron, and $\varepsilon_{o}$ is the permittivity in vacuum. Figures 5.(a) and (b) show the profile of the plasma frequency and the refractive index, where $\omega_{p o}(t)$ and $n_{0}(t)$ are the plasma frequency and the refractive index, respectively at $z=h_{0}$ where the electron density is maximum.

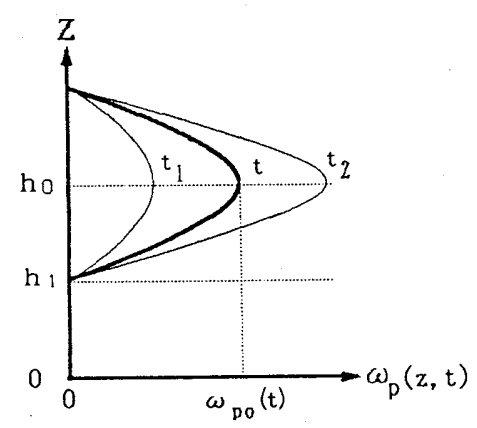

(a)

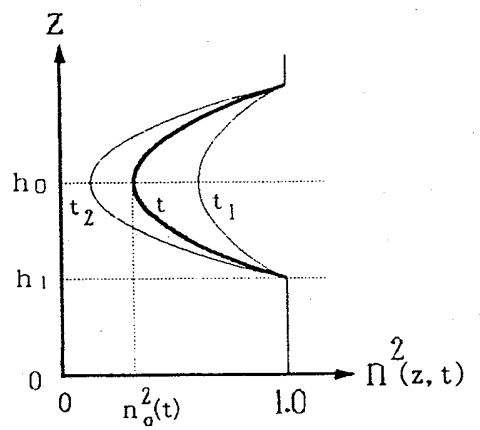

(b)

Fig.5. Profiles of plasma frequency (a) and refractive index (b). 
Applying a geometrical optics approximation to our model of the ionosphere, we have the following Eikonal equation (Tyras, 1969; Ishimaru, 1991),

$$
\left(\frac{\partial S}{\partial x}\right)^{2}+\left(\frac{\partial S}{\partial z}\right)^{2}=n^{2}(z, t)
$$

where $S$ means the length along the ray path. Applying the variable separation method of $S=f_{1}(x)+$ $f_{2}(z)$ to the above equation, we can obtain the solution

$$
S=a x \pm \int_{0}^{z} \sqrt{n^{2}(x, z)-a^{2}} d z
$$

where $\mathrm{a}$ is the integral constant. To determine a, we can apply Fermat's principle, $\delta \mathrm{S} / \delta \mathrm{a}=0$ to the equation (8), then we have the following equations,

$$
\begin{aligned}
& x= \pm \int_{0}^{z} \frac{a}{\sqrt{n^{2}(x, z)-a^{2}}} d z \\
& a=n(z, t) \sin \theta(z, t)=n_{a} \sin \theta_{a}(t)=n\left(z_{m}, t\right)
\end{aligned}
$$

where $\mathrm{n}_{\mathrm{a}}$ is the refractive index in the air, $\theta(z, t)$ is the angle between $z$-axis and the ray path, $\theta_{\mathrm{a}}$ is the angle of incidence, and $z_{\mathrm{m}}$ is the height of the turning point.

Substituting Eqs. (3) through (4) and (10) to Eq.(9) and calculating the integral after dividing into two parts as $z=0$ to $h_{1}$ and $z=h_{1}$ to $z$, we can obtain Eq.(11). This is the equation for the propagation path. We can plot the ray path taking $x$ as a function of $z$.

$$
\begin{aligned}
x=h_{1} \tan \theta_{a}(t) & +\sin \theta_{a}(t) \frac{\omega \sqrt{1-Y_{L}}\left(h_{0}-h_{1}\right)}{\omega_{p_{O}}(t)} \\
& \times \log _{e} \frac{\left(h_{0}-h_{1}\right)+\sqrt{\left(h_{0}-h_{1}\right)^{2}-\left(h_{0}-z_{m}\right)^{2}}}{\left(h_{0}-z\right)+\sqrt{\left(h_{0}-z\right)^{2}-\left(h_{0}-z_{m}\right)^{2}}}
\end{aligned}
$$

where $\theta_{a}$ is the incident angle to the ionosphere. Then, applying the conoition of $z=z_{m}$ when $\mathrm{x}=\mathrm{D} / 2$ to Eq.(11), we can obtain Eq.(12).

$$
\begin{aligned}
\frac{D}{2}=h_{1} \tan \theta_{a}(t) & +\sin \theta_{a}(t) \frac{\omega \sqrt{1-Y_{L}}\left(h_{0}-h_{1}\right)}{\omega_{p O}(t)} \\
& \times \log _{e} \frac{\left(h_{0}-h_{1}\right)+\sqrt{\left(h_{0}-h_{1}\right)^{2}-\left(h_{0}-z_{m}\right)^{2}}}{h_{0}-z_{m}}
\end{aligned}
$$

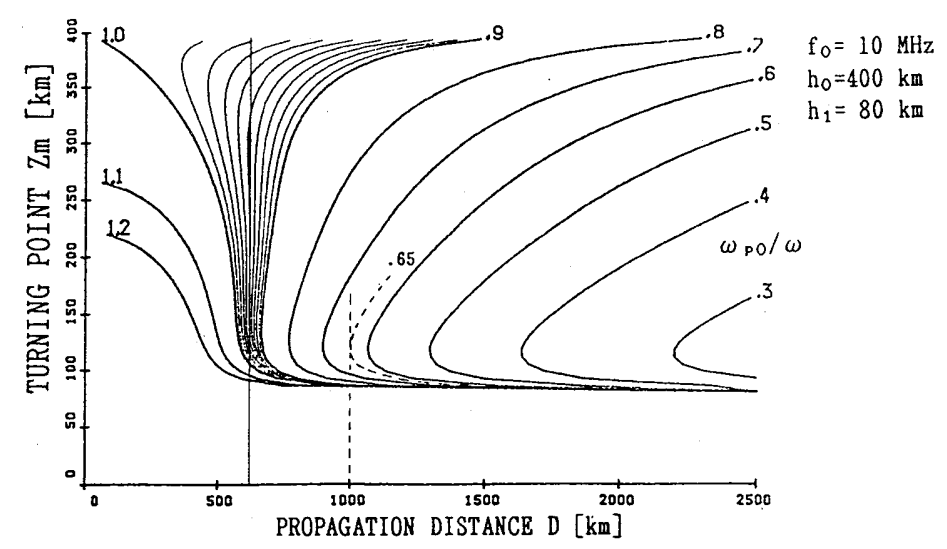

(a) 


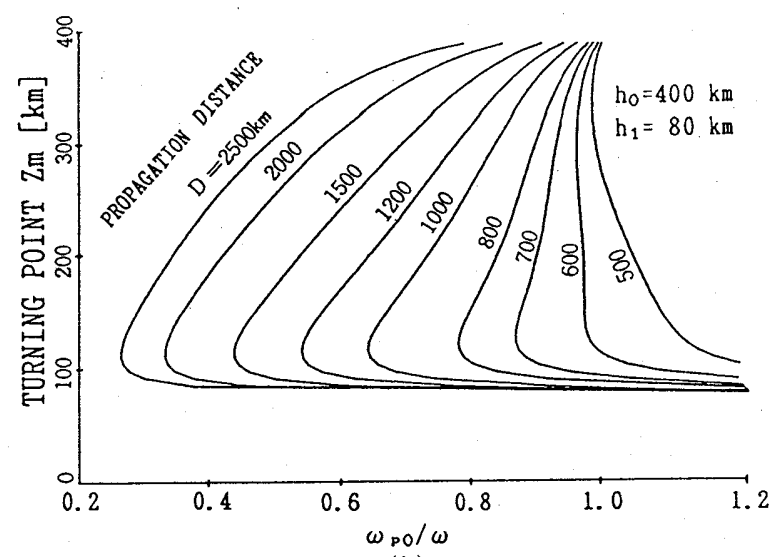

(b)

Fig.6. Height of turning points as a function of propagation distance with the plasma frequency as a parameter (a) and as a function of plasma frequency with the propagation distance as a parameter (b).

Solving Eq.(12) by means of Muller's iteration method (DEC, 1981), we can seek the height of turning points $z_{m}$. Then, we can plot the ray path substituting $z_{m}$ again into Eq.(11). Figure 6(a) gives the turning points obtained from Eq.(12) as a function of the propagation distance with the plasma frequency $\omega_{p o}(t)$ as a parameter. Figure $6(\mathrm{~b})$ gives the turning points as a function of plasma frequency with the propagation distance $D$ as a parameter.

It is seen from Fig.6(a) that at a distance, say $1000 \mathrm{~km}$, the wave turning point does not exist when the plasma frequency $\omega_{\text {po }}$ is low, say less than $6.5 \mathrm{MHz}$. But the wave turning point does appear once for the plasma frequency of $6.5 \mathrm{MHz}$ indicated by the dotted line. Then the two turning points appear as $\omega_{\text {po }}$ increases. As $\omega_{\text {po }}$ further increases, one of the turning points falls while the other point rises.

The calculated ray paths are shown in Fig.7. When the electron density increases, an ionospheric reflected-wave appears suddenly at a level in the ionosphere as shown by a dashed line. This wave makes the nose of $C$-shaped trace. Then a ray called upper ray goes up as indicated by an upward arrow, while another ray called lower ray goes down as indicated by a downward arrow. The path length in the phase space becomes shorter for the upper ray than for the lower ray because the phase velocity for the upper ray becomes faster than the lower ray. Then it is interpreted that the upper ray makes the upper part of the C-shaped trace, while the lower ray makes the lower part.

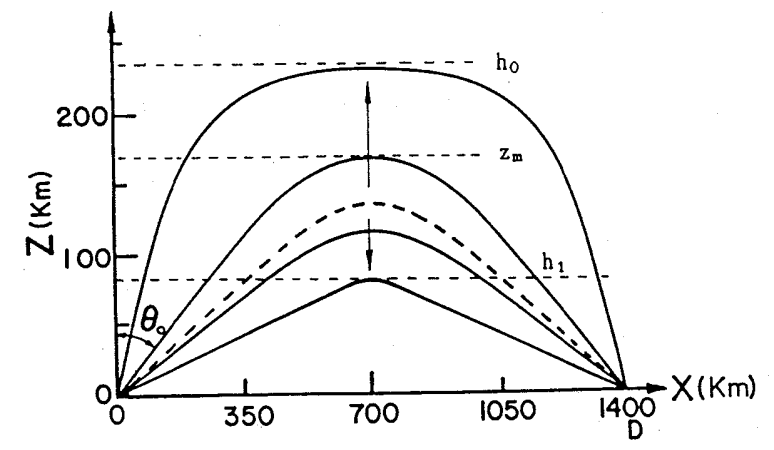

Fig. 7. Ray paths calculated by using $D=1400 \mathrm{~km}, \mathrm{~h}_{1}=80 \mathrm{~km}$, and $\mathrm{h}_{\mathrm{o}}=240 \mathrm{~km}$. The path for the nose point of the trace indicated by the dashed line, and two upper rays and two lower rays are drawn by calculating Eq.(11) by solid line.

As the wave goes up to higher levels, the wave velocity becomes higher than the wave along lower paths, then the wave along higher paths has more deviation in Doppler effect. It can also be seen in Fig.6(a) that three or four turning points can exist at a distance near $600 \mathrm{~km}$. These facts tell us that the $Z$-shaped trace might be made from the three turning points and the $\varepsilon$-shaped trace from the four turning points. These might be observed at our station, Nankoku where is 
located at $617 \mathrm{~km}$ from the JJY station. We will discuss the frequency variation obtained from three turning points which may make the $Z$-shaped traces and four turning points which may make the $\varepsilon$-shaped traces in the next paper. Two turning points in Fig. 6 lead to the appearance of the $\mathrm{C}$-shaped and the inversed C-shaped characteristic of Doppler deviation.

\section{Derivation of Doppler deviation}

The Doppler deviation $\triangle \mathrm{f}$ is calculated from the following equation, using Eqs. (8) and (10), since it is obtained by differentiating the path length $S$ (Davies, 1965):

$$
\Delta f(t)=-\frac{2 f_{0}}{c} \frac{d}{d t}\left\{D \sin \theta_{a}(t)+\int_{0}^{z_{m}} \sqrt{n^{2}(z, t)-\sin ^{2} \theta_{a}(t)} d z\right\}
$$

Applying Eqs.(4) and (5) to Eq.(13) and using Eq.(10), we obtain the following equations.

$$
\begin{aligned}
\Delta f(t) & =\frac{f_{0}}{c} \frac{d}{d t}\left[\frac { \omega _ { p O } ( t ) } { \omega ( h _ { 0 } - h _ { 1 } ) } \left\{\left(h_{0}-z_{m}\right)^{2} l_{0} \frac{\left(h_{0}-h_{1}\right)+\sqrt{\left(h_{0}-h_{1}\right)^{2}-\left(h_{0}-z_{m}\right)^{2}}}{h_{0}-z_{m}}\right.\right. \\
& \left.\left.-\left(h_{0}-h_{1}\right) \sqrt{\left(h_{0}-h_{1}\right)^{2}-\left(h_{0}-z_{m}\right)^{2}}\right\}-2 D \sin \theta_{a}(t)-2 h_{1} \cos \theta_{a}(t)\right] \\
& \sin \theta_{a}(t)=\sqrt{1-\frac{\omega_{p O}^{2}(t)}{\omega}\left\{1-\left(\frac{h_{0}-Z}{h_{0}-h_{1}}\right)^{2}\right\}}
\end{aligned}
$$

Thus, the Doppler deviation $\triangle \mathrm{f}$ is obtained as a function of time by obtaining $z_{m}$ from Eq.(12), by substituting it in Eq.(14), and by numerically processing a differential operation, if the functional form of $\omega_{p o}(t)$ is given.

\section{Estimation of the maximum plasma frequency, $\omega_{\text {po }}(t)$}

The maximum plasma frequency $\omega_{\text {po }}(t)$ at ionospheric reflection points can be obtained by reading the f-plot curve in Ionospheric Data in Japan (Communication Research Laboratory, 1981). Figure 8(a) shows an example of the $\mathrm{f}-$ plot curves and Fig. 8 (b) shows a magnification of the variation in $f_{p o}(t)=\omega_{p o}(t) /(2 \pi)$ from 5:06 to 8:00 JST. In Fig. $8(b)$, two pairs of curves for ordinary and extraordinary waves are indicated. The one pair of curves is for the data obtained at Kokubunji located north of Nankoku and the other at Yamagawa located south of Nankoku. Three time axis' are referred to the times of sunrise at the ionospheric reflecting points of JJY, BPV, and BPM.

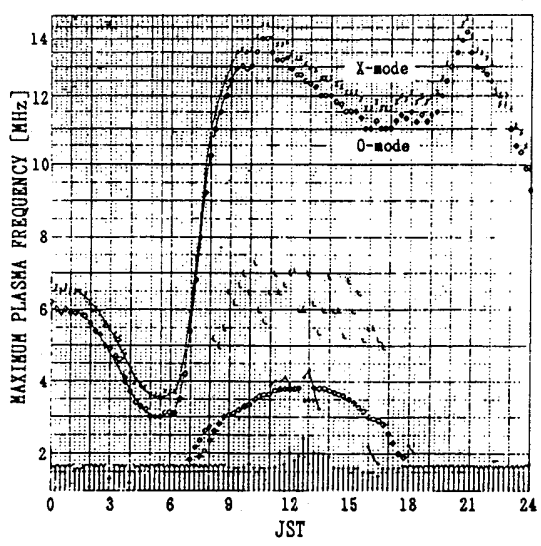

(a)

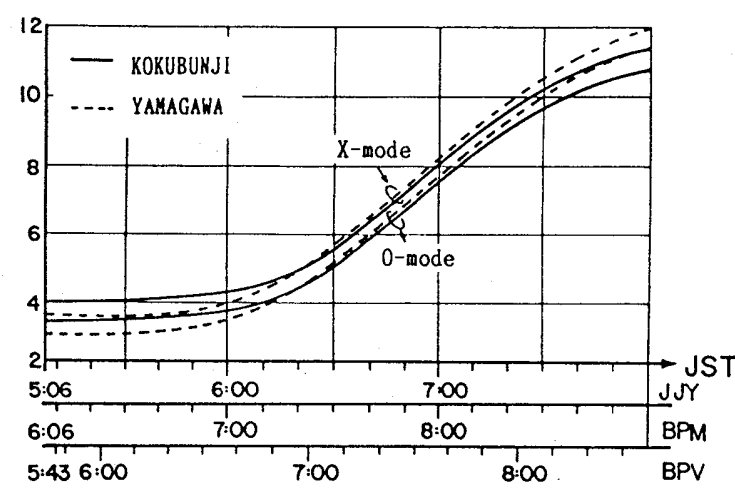

(b)

Fig.8. An example of f-plot curve. (a) February 15, 1981 at Yamagawa. (b) Magnification of the data from 5:06 to 8:00 JST.

To obtain the plasma frequency at the turning point of each wave from the f-Plot curve, we must examine the time difference in sunrise between the reflection point at the ionosphere and the f-Plot measuring point, Kokubunji or Yamagawa. After that, we must compensate for the time 
difference between the turning points of each wave. The sunrise time $\mathrm{T}$ is obtained from Eqs.(16) and (17) when latitude $\phi$ and longitude $\beta$ at the wave turning point are given (Watanabe, 1969),

$$
\begin{gathered}
\mathrm{T}=12-\frac{24}{360} \cos ^{-1}\left\{\cos \left(90^{\circ}+\xi\right) \sec \phi \sec \delta-\tan \phi \tan \delta\right\}-\mathrm{E}+\frac{4}{60}\left(\beta_{0}-\beta\right) \\
\xi=\cos ^{-1}\left(\frac{\mathrm{R}}{\mathrm{R}+\mathrm{z}_{\mathrm{m}}}\right), \quad \delta=23.43 \sin \eta
\end{gathered}
$$

where $\mathrm{E}$ is the time difference, $\beta_{\mathrm{o}}$ is the longitude of the standard meridian, $\mathrm{R}$ is the earth's radius, $z_{m}$ is the height of the reflection point at the ionosphere, and $\eta$ is the zodiacal range angle from the vernal equinoctial point. The calculated results by using Eq.(16) are listed in Table 1. The times of sunrise on February 15, 1981 were obtained by using the values of $R=6,370 \mathrm{~km}$, $\mathrm{E}=0.24$ hours, $\delta=-13 \mathrm{deg}$, and $\mathrm{z}_{\mathrm{m}}$, height of the ionospheric reflection point as parameters.

Table 1. Locations of the ionospheric reflection points of JJY, BPV, and BPM, and Kokubunji and Yamagawa, and times of sunrise at four turning

\begin{tabular}{|c|c|c|c|c|c|}
\hline \multicolumn{2}{|c|}{ Location } & 0 & 100 & 200 & 400 \\
\hline \multirow{3}{*}{ 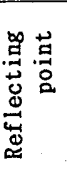 } & JJY $\left(\begin{array}{cc}136^{\circ} & 43^{\prime} \mathrm{E} \\
34^{\circ} & 54^{\circ} \mathrm{N}\end{array}\right)$ & $6: 45$ & $5: 54$ & $5: 34$ & $5: 06$ \\
\hline & $\mathrm{BPV}\left(\begin{array}{r}127^{\circ} \\
32^{\circ}\end{array} \frac{28^{\prime}}{31^{\prime}} \mathrm{E}\right)$ & $7: 18$ & $6: 29$ & $6: 09$ & $5: 43$ \\
\hline & BPM $\left(\begin{array}{cc}121^{\circ} & 43^{\circ}, \mathrm{B} \\
34^{\circ} & 52^{\prime}\end{array}\right)$ & $7: 45$ & $6: 54$ & $6: 34$ & $6: 06$ \\
\hline \multirow{2}{*}{ 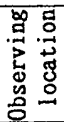 } & Koku $\left(\begin{array}{cc}139^{\circ} & 29^{\prime}, \mathrm{B} \\
35^{\circ} & 42^{\prime}\end{array}\right)$ & $6: 35$ & $5: 44$ & $5: 24$ & $4: 56$ \\
\hline & Yama $\left(\begin{array}{rr}130^{\circ} & 37^{\prime}, \mathrm{B} \\
31^{\circ} & 12^{\prime}\end{array}\right)$ & $7: 04$ & $6: 16$ & $5: 57$ & $5: 30$ \\
\hline
\end{tabular}
points on February 15, 1981 calculated by using $\mathrm{E}=0.24 \mathrm{hrs}$. and $\delta=-13.0 \mathrm{deg}$.

The sunrise time at the ionospheric reflecting points of waves from JJY, BPV, and BPM, and of waves radiated vertically from Kokubunji and Yamagawa are plotted up to $600 \mathrm{~km}$ in the ionosphere in Fig.7.

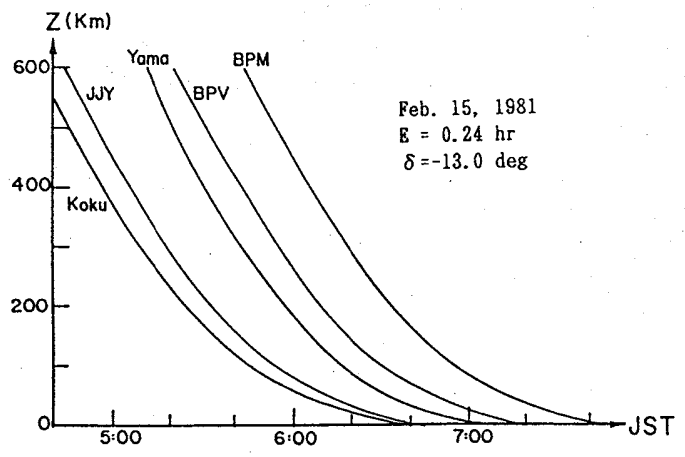

Fig.7. The sunrise time at the ionospheric reflecting points of waves from JJY, BPV, and BPM, and of waves radiated vertically from Kokubunji and Yamagawa.

The value of $\omega_{p o}(t)=2 \pi f_{p o}(t)$ at the reflection points can be obtained by proportionally dividing the values between the two curves labeled Kokubunji and Yamagawa in Fig. $6(\mathrm{~b})$ according to the latitudes of the reflecting points. The value of $\Delta f(t)$ is calculated by substituting the value of $\omega_{\text {po }}(\mathrm{t})$ to Eqs. (15) and (14) in the order named. A method of approximation to obtain $\omega_{\text {poo }}(t)$ from the curves of Fig. $6(\mathrm{~b})$ by cubic equations is employed for the numerical calculation of Eq.(14).

6. Moving down effect of the ionosphere.

The sunrise point moves from the upper layer to the lower layer of the ionosphere as seen in Fig.7, so that the ionization by ultraviolet radiation is considered to begin also from the upper layer. Thus, the bottom level $h_{1}$ and the maximum density height $h_{0}$ of the ionosphere shown in 
Figs. 4 and 5 are considered to move down also at the sunrise. In the ionosphere in winter, $F$ layer develops first, followed by $E$ layer, and then by $D$ layer; these layers do not develop into a single layer. However, we assume both $h_{1}$ and $h_{0}$ to move down at the same speed in the first order approximation on the premise that the transfer from $F$ layer to $E$ layer occurs continuously. Thus, Eq.(14) is processed as follows.

$$
h_{1}=h_{11}-\Delta h \exp \left(-\frac{t-t_{0}}{\alpha}\right), \quad h_{0}=h_{00^{-}}-\Delta h \exp \left(-\frac{t-t_{0}}{\alpha}\right)
$$

The value of $\alpha$ is determined by comparing the calculated values with the measured values after repeated trial and errors.

7. Comparison between the calculated and the measured values

Figures 8(a) and (b) show the calculated results of Eq.(14). In the measured values of Doppler deviation as shown in Fig. 3, the $5 \mathrm{MHz}$ wave was received at the same time as the $10 \mathrm{MHz}$ wave for calibrating $\Delta f$ axis. The analysis revealed that the point of $h_{11}=300 \mathrm{~km}, h_{0 o}=400 \mathrm{~km}, \Delta h=200 \mathrm{~km}$, and $\alpha=40 \mathrm{~min}$ is suitable for the data in Fig.3(a) observed on February 15, 1981. The values of 46 min from JJY, 9.0 min from BPV, and -14.0 min from BPM were selected for $t_{o}$, considering the time difference in Fig. 7 which shows the sunrise at the reflecting point of each wave.

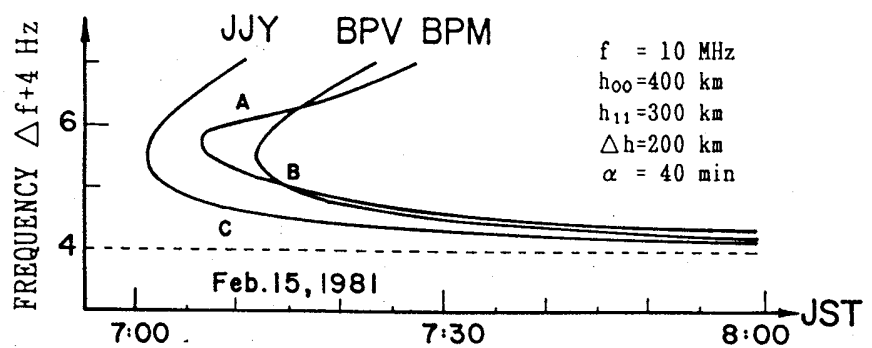

(a)

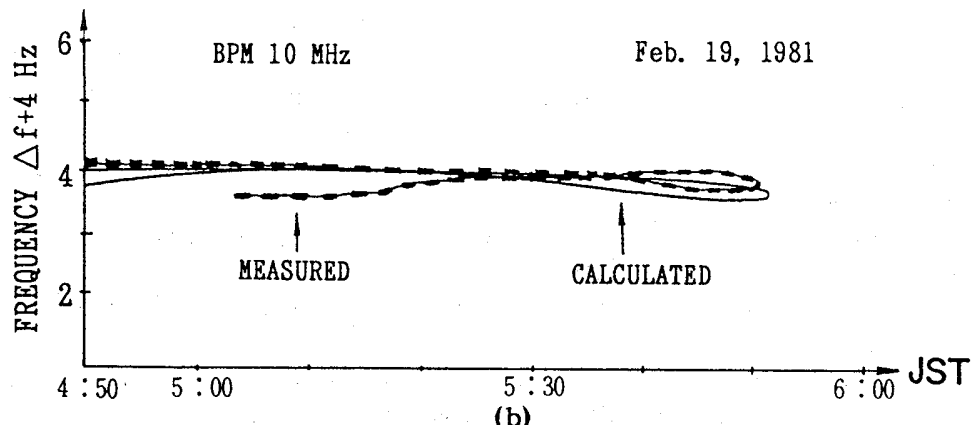

Fig.8. Calculated Results. (a) Simulation for measured C-shaped traces in Fig. 3(a). (b) Simulation for $\infty$-shaped trace in Fig.3(d).

The calculated results of simulation for $\mathrm{C}$-shaped and $\infty$-shaped traces are shown in Fig. 8 . It is seen by comparison of Fig. 8 with Fig. 3 that the calculated carves coincide considerably well with the measured curves. The coincidence is summarized as follows:

1) A deep fading is caused mainly by the Doppler deviation which shows the $\mathrm{C}$-shaped and the inversed $\mathrm{C}$-shaped traces on the sonagram.

2) The C-shaped curves appear for JJY first, for BPM next, and for BPV last at about $6 \mathrm{~Hz}$ in Fig.8(a). These features are similar to the measured ones in Fig.3(a). In other words, the $\mathrm{C}$-shaped variation appear earlier for the wave from BPM than from BPV, for the sun rises at the $\mathrm{BPM}$ reflection area later than that from BPV. In addition, curve A for BPM shows a sharper bend than the other two curves.

3) Curves of BPV and BPM cross each other at point B. (Crossing each other twice above and below $6 \mathrm{~Hz}$ in calculation as seen in Fig.8(a).), and slow curving occurs near point $\mathrm{C}$. 
4) If the plasma frequency decreases slowly, the Doppler deviation shows a U-turn trace, and if plasma frequency oscillates slowly, the trace shows the $\infty$-shaped.

5) For linear and exponential profiles of electron density, similar traces are obtained.

6) If not considering the moving down effect of the ionosphere, we get similar traces but features of the $\mathrm{C}^{-}$and the inversed $\mathrm{C}$-shaped curves are somewhat different from the measured ones.

8. Concluding remarks

We discussed a calculation method with only a parabolic profile of the ionospheric electron density. We tried to calculate with other profiles like linear and exponential profiles given by Kitamura et al. (1979), but the parabolic profile explained best our observed traces. Calculated ray paths and C-shaped traces calculated by Eq.(14) with fixed $h_{1}$ and $h_{2}(\Delta h=0)$ agree with the results calculated by Kitamura et al. (1980).

When the ionosphere is calm, the variation in electron density depends only on the solar radiation. The plasma frequency was assumed to change in the same manner on the same latitude with a time delay by the earth's rotation. The subject of the future study will be an analysis of more complicated shape of the traces such as Z-shaped and $\varepsilon$-shaped characteristics appearing at the sunrise.

\section{Acknowledgments}

The authors wish to express their heartfelt gratitude to Prof. Tohru Ogawa at Upper Atmosphere Research Laboratory, Kyoto University, Prof. Takeo Yoshino, and Mr. Ichiro Tomizawa, Department of Applied Electronic Engineering, University of Telecommunications, who provided us with documents on Doppler observation including a circuit diagram of the real-time analyzer and Planning Department of Communications Research Laboratory who supplied us with the Ionospheric Data in Japan, for their support and guidance that enabled us to conduct the study reported here. We wish to thank Prof. Taiichi Kitamura and Dr. Masasi Takefu for their kindness and useful discussion on $\mathrm{C}$-shaped characteristics, and Mr. Eiji Nishimori for improving the radio wave receiver, Mr. Tetsuro Tsuzuki, and Mr. Tshuyoshi Mizukuchi for promoting the numerical calculation. Thanks are also due to Hitachi Communication Systems, Incorporated for their support in part.

\section{References}

Budden, K. G., Radio Waves in the Ionosphere, Cambridge University Press, Cambridge, pp. 542, 1961a.

Davies, K., Ionospheric Radio Propagation, National Bureau of Standards Monograph 80, Washington, D.C., pp. 470, 1965.

DEC, Scientific Subroutine Programer's Reference Manual, Degital Equipment Corporation, Technical Document Center, Illinois, 3-45, 1981.

Ishimaru, A., Electromagnetic Wave Propagation, Radiation, and Scattering, Prentice-Hall International, Inc., pp.637, 1991.

Kitamura, T. and M. Takefu, HF Doppler observation and change in electron density with time, HF Doppler Study Report, 1, 5-9, Oct., 1979. (in Japanese)

Kitamura, T. and M. Takefu, The sunrise effect (Part 2), HF Doppler Study Report, 2, 12-16, Dec., 1980. (in Japanese)

Radio Research Laboratories (now Communication Research Laboratories), Ionospheric Data in Japan, Ministry of Posts and Telecommunications, 104-105, Feb.,1981.

Takefu, M. and T. Kitamura, The Foundmental theory on HF Doppler, HF Doppler Study Report, 1, 92-159, Apr., 1982. (in Japanese)

Tyras, G., Radiation and Propagation of Electromagnetic Waves, Academic Press, New York, pp. 368, 1969.

Yano, S. A. Ishimaru, and K. Imai, Changes in the ionosphere caused by the sunrise, Bulletin of Kochi National College, 18, 54-58, Aug. 1982a. (in Japanese)

Yano, S. and A. Ishimaru, A mechanism to make C-shaped form and focusing effect of waves, HF Doppler Study Report, 13-17, April 1982b. (in Japanese)

Yano, S. and A. Ishimaru, The 8-shaped trace and fading phenomena in Doppler deviation, Bulletin of Kochi National College, 79-84, Aug. 1983. (in Japanese)

Watanabe, T., Mathematical Astronomy, Kohseisha-Kohseikaku Press, pp. 410, 1969. (in Japanese)

(Received December 1, 1992; Revised December 29, 1992; Accepted January 5, 1993.) 